\title{
Digital health approaches for cardiovascular diseases prevention and management: lessons from preliminary studies
}

Recent advances in digital health technologies including electronic and mobile health platforms (eHealth and mHealth), telemedicine, wearable devices, sensors and artificial intelligence (AI) provide opportunities to improve access to and delivery of healthcare (1). Digital health services currently employ the use of digital technologies for the provision of health education and awareness (i.e., text messaging), remote monitoring and support (i.e., telerehabilitation), disease prediction (i.e., AI), and vital signs monitoring (i.e., wearable devices) (2). However, digital technologies have also been used as diagnostic tools-for example, machine learning and deep learning approaches for the detection of diabetic retinopathy and skin cancers (3). Along with the large datasets generated by electronic health records and medical devices, the global market for digital health has increased steadily over the past few years and projected to reach from USD \$106 billion in 2019 to USD \$639.4 billion in 2026 (4). These big data provide opportunities to understand disease trends, gain insights in patients' health, better predict future health outcomes and support individual care.

Cardiovascular diseases (CVD) has been at the forefront of digital health innovations. A systematic review and metaanalysis of 51 articles assessing the benefit of digital health on CVD showed that digital health interventions significantly reduced CVD outcomes (Relative Risk 0.61, 95\% CI, 0.46-0.80) with concomitant reductions in weight ( $-2.77 \mathrm{lb},-4.49$ to $-1.05 \mathrm{lb})$ and body mass index $\left(-0.17 \mathrm{~kg} / \mathrm{m}^{2},-0.32\right.$ to $\left.-0.01 \mathrm{~kg} / \mathrm{m}^{2}\right)$ compared with usual care (5). In the six studies, 10-year risk percentages were also significantly improved $(-1.24 \% ;-1.73 \%$ to $-0.76 \%)$. A recent individual patient data meta-analysis reported that text messaging program had a modest impact on blood pressure and body mass index (6). A systematic review of 14 articles assessing the cost-effectiveness of digital health interventions for CVD showed that all studies were cost-effective (7). Digital technologies offer significant opportunities for CVD prevention by promoting lifestyle change and adherence to healthy behaviours (8), early diagnosis, individualised management/supportive care and clinical decision support (9). Given the importance of technologies for the prevention and management of CVD, this special series is focused on recent developments in digital health for CVD. Five papers are presented in the series and are summarised below.

\section{A mHealth tele-imaging platform using WhatsApp}

Several recent studies have demonstrated the use of mHealth as a tele-imaging platform in dermatology (10), oral medicine (11), pathology (12) and radiology (13). In this special series, Giansanti et al. reported on the exchange of hemodynamic images in cardiology using WhatsApp with a client-server-architecture. The WhatsApp system was pilot tested in a clinical setting using five dynamic images using standardised hospital procedures. The system was evaluated by five experts in interventional radiology who ranked the system high for easy to use, utility and speed, and average for image quality and technology. The use of WhatsApp for exchanging cardiology images might be useful in future telemedicine practise.

\section{Smartphone-based survey on AI in cardiology}

AI is a promising tool in cardiology, and offers several utilities including automated image interpretation, data extraction and quality control, and clinical risk prediction (14). Giansanti et al. investigated the role of AI in cardiology among 30 cardiologists. Their results indicate that cardiologists have a high desire to invest in AI and use AI in several fields of cardiology, but have low confidence in the use of AI and quality control procedures. In addition, cardiologists have a strong belief that ethical issues are hampering the diffusion of AI. Despite the potential of AI, it requires further refinement and evaluation before it can be used in cardiology practice.

\section{Self-service health check-up stations for screening atrial fibrillation (AF)}

$\mathrm{AF}$ is one of the most common causes of ischemic stroke, leading to premature deaths and disabilities. Early detection of 
$\mathrm{AF}$ and treatment could prevent the majority of stroke events. To detect clinically significant AF, considerable amounts of monitored data may be required (15). In a review article, Boulos and Haywood introduce the concept and potential benefits of opportunistic AF screening and detection in a community setting using the latest generation of affordable digital ECG capture and interpretation solutions integrated into easy-to-use "self-service health check-up stations". Future studies are needed to demonstrate the effectiveness and cost-effectiveness of such check-up stations for AF detection.

\section{mHealth intervention for dietary behaviour}

mHealth programs have shown to improve health outcomes in diabetes (16), CVD (6,17-19), chronic obstructive pulmonary disease (20), depression (21), promote physical activity and dietary behaviour (22-24). However, mHealth programs for dietary behaviour for diabetes in low- and middle-income countries (LMICs) are scares. In a post-hoc analysis from a randomised controlled trial (RCT), Islam et al. described the effects of a 6-month text messaging lifestyle program on dietary behaviour in people with type 2 diabetes in Bangladesh. In a previous study, the authors reported that the program was effective and costeffective for diabetes care $(25,26)$, however the post-hoc analysis showed no significant effect on dietary behaviour. Targeting dietary behaviours in diabetes mHealth interventions could possibly further improve glucose control. With increasing prevalence of diabetes and hypertension (27-30) and mobile phone use (31), mHealth can be an adjunct approach to support patient care in LMICs (32). Further studies with targeted messages for dietary behaviour are recommended.

\section{A clinical decision support system (CDSS) for non-communicable diseases (NCDs)}

LMICs have the highest prevalence and death rates of NCDs, including CVDs $(33,34)$. Challenges in LMICs for NCD programs include shortages of qualified practitioners, lack of competencies among the health workforce, lack of proper referral and poor health infrastructures. Task shifting by Community Health Workers (CHWs) has been proven to be a practical approach for many health conditions (35). Zaman and colleagues describe the protocol for an RCT testing a CDSS and the associated community-based management program for people with NCDs in India. The study will examine whether the program is user-friendly, easy-to-comprehend, easy-to-deliver, workflow-oriented, and comprehensive and result in better management of hypertension and co-morbidities than usual care.

\section{The way forward}

This special series on Digital Health for Cardiovascular Disease highlights the potential application of digital health technologies for the prevention and management of CVD. Collectively, these studies highlight the potential of smartphone apps (e.g., WhatsApp), text-messaging approaches, as well as AI and decision instruments (e.g., CDSS) to enhance CVD management. Nevertheless, numerous challenges hinder the adoption of these technologies, including data quality and robustness, patient safety, ease of use, privacy concerns, and accessibility. Research is needed to advance our understanding of how new digital solutions (e.g. wearable devices, social media) can support prevention and management of CVD (36,37), as well as to improve our understanding of how to implement effective programs at scale, and into everyday practice. As healthcare continues to advance, we need to ensure that we remain conscious of our ultimate goal to support the wellbeing of people with CVD to lead a healthier and productive life.

\section{Acknowledgments}

Funding: None.

\section{Footnote}

Provenance and Peer Review: This article was commissioned by the editorial office, mHealth for the series "Digital Health for Cardiovascular Disease". The article did not undergo external peer review. 
Conflicts of Interest: Both authors have completed the ICMJE uniform disclosure form (available at http://dx.doi.org/10.21037/ mHealth-2020-6). The series "Digital Health for Cardiovascular Disease" was commissioned by the editorial office without any funding or sponsorship. Sheikh Mohammed Shariful Islam served as the unpaid Guest Editor of the series and serves as an unpaid editorial board member of the mHealth from May 2015 to Apr 2021. Ralph Maddison served as the unpaid Guest Editor of the series. Sheikh Mohammed Shariful Islam reports grants from National Heart Foundation of Australia, grants from NHMRC, other from World Health Organization, other from World Heart Federation, outside the submitted work. Ralph Maddison reports grants from National Heart Foundation of Australia, grants from NHMRC, outside the submitted work. The authors have no other conflicts of interest to declare.

Ethical Statement: The authors were accountable for all aspects of the work in ensuring that questions related to the accuracy or integrity of any part of the work were appropriately investigated and resolved.

Open Access Statement: This is an Open Access article distributed in accordance with the Creative Commons AttributionNonCommercial-NoDerivs 4.0 International License (CC BY-NC-ND 4.0), which permits the non-commercial replication and distribution of the article with the strict proviso that no changes or edits are made and the original work is properly cited (including links to both the formal publication through the relevant DOI and the license). See: https://creativecommons.org/licenses/by-nc$\mathrm{nd} / 4.0 /$.

\section{References}

1. Panch T, Pearson-Stuttard J, Greaves F, et al. Artificial intelligence: opportunities and risks for public health. Lancet Digit Health 2019;1:e13-4.

2. Frederix I, Caiani EG, Dendale P, et al. ESC e-Cardiology Working Group Position Paper: Overcoming challenges in digital health implementation in cardiovascular medicine. Eur J Prev Cardiol 2019;26:1166-77.

3. Sharma A, Harrington RA, McClellan MB, et al. Using Digital Health Technology to Better Generate Evidence and Deliver Evidence-Based Care. J Am Coll Cardiol 2018;71:2680-90.

4. Mahajan S. Digital Health Market Size, Growth and Industry Trends Report, 2026. 2020. Available online: https://www. gminsights.com/industry-analysis/digital-health-market

5. Widmer RJ, Collins NM, Collins CS, et al. Digital Health Interventions for the Prevention of Cardiovascular Disease: A Systematic Review and Meta-analysis. Mayo Clin Proc 2015;90:469-80.

6. Shariful Islam SM, Farmer AJ, Bobrow K, et al. Mobile phone text-messaging interventions aimed to prevent cardiovascular diseases (Text2PreventCVD): systematic review and individual patient data meta-analysis. Open Heart 2019;6:e001017.

7. Jiang X, Ming WK, You JH. The cost-effectiveness of digital health interventions on the management of cardiovascular diseases: systematic review. J Med Internet Res 2019;21:e13166.

8. Khan N, Marvel FA, Wang J, et al. Digital Health Technologies to Promote Lifestyle Change and Adherence. Curr Treat Options Cardiovasc Med 2017;19:60.

9. Bhavnani SP, Parakh K, Atreja A, et al. 2017 Roadmap for Innovation-ACC Health Policy Statement on Healthcare Transformation in the Era of Digital Health, Big Data, and Precision Health: A Report of the American College of Cardiology Task Force on Health Policy Statements and Systems of Care. J Am Coll Cardiol 2017;70:2696-718.

10. Williams V, Kovarik C. WhatsApp: An innovative tool for dermatology care in limited resource settings. Telemed J E Health 2018;24:464-8.

11. Petruzzi M, De Benedittis M. WhatsApp: a telemedicine platform for facilitating remote oral medicine consultation and improving clinical examinations. Oral Surg Oral Med Oral Pathol Oral Radiol 2016;121:248-54.

12. Giansanti D, Castrichella L, Giovagnoli MR. The design of a health technology assessment system in telepathology. Telemed J E Health 2008; 14:570-5.

13. Giansanti D. WhatsApp in mHealth: an overview on the potentialities and the opportunities in medical imaging. mHealth 2020;6:19.

14. Kilic A. Artificial Intelligence and Machine Learning in Cardiovascular Health Care. Ann Thorac Surg 2020;109:1323-9. 
15. Sanna T, Diener HC, Passman RS, et al. Cryptogenic stroke and underlying atrial fibrillation. N Engl J Med 2014;370:2478-86.

16. Kitsiou S, Paré G, Jaana M, et al. Effectiveness of mHealth interventions for patients with diabetes: an overview of systematic reviews. PloS One 2017;12:e0173160.

17. Chow CK, Ariyarathna N, Islam SMS, et al. mHealth in cardiovascular health care. Heart Lung Circ 2016;25:802-7.

18. Maddison R, Rawstorn JC, Islam SMS, et al. mHealth interventions for exercise and risk factor modification in cardiovascular disease. Exerc Sport Sci Rev 2019;47:86.

19. Chow CK, Redfern J, Hillis GS, et al. Effect of lifestyle-focused text messaging on risk factor modification in patients with coronary heart disease: a randomized clinical trial. JAMA 2015;314:1255-63.

20. Vorrink SN, Kort HS, Troosters T, et al. Efficacy of an mHealth intervention to stimulate physical activity in COPD patients after pulmonary rehabilitation. Eur Respir J 2016;48:1019-29.

21. Shariful Islam SM, Chow CK, Redfern J, et al. Effect of text messaging on depression in patients with coronary heart disease: A sub study analysis from the TEXT ME randomised controlled trial. BMJ Open 2019;9:e022637.

22. Spring B, Pellegrini C, McFadden H, et al. Multicomponent mHealth intervention for large, sustained change in multiple diet and activity risk behaviors: the make better choices 2 randomized controlled trial. Journal of medical Internet research 2018;20:e10528.

23. Vandelanotte C, Müller AM, Short CE, et al. Past, present, and future of eHealth and mHealth research to improve physical activity and dietary behaviors. J Nutr Educ Behav 2016;48:219-28.e1.

24. Martin SS, Feldman DI, Blumenthal RS, et al. mActive: a randomized clinical trial of an automated mHealth intervention for physical activity promotion. J Am Heart Assoc 2015;4:e002239.

25. Shariful Islam SM, Niessen LW, Ferrari U, et al. Effects of Mobile Phone SMS to Improve Glycemic Control Among Patients With Type 2 Diabetes in Bangladesh: A Prospective, Parallel-Group,Randomized Controlled Trial. Diabetes Care 2015;38:e112-3.

26. Islam SMS, Peiffer R, Chow CK, et al. Cost-effectiveness of a mobile-phone text messaging intervention on type 2 diabetes-A randomized-controlled trial. Health Policy Technol 2020;9:79-85.

27. Biswas T, Islam A, Rawal LB, et al. Increasing prevalence of diabetes in Bangladesh: a scoping review. Public health 2016;138:4-11.

28. Islam SMS, Mainuddin A, Islam MS, et al. Prevalence of risk factors for hypertension: A cross-sectional study in an urban area of Bangladesh. Glob Cardiol Sci Pract 2015;2015:43.

29. Shariful Islam SM, Lechner A, Ferrari U, et al. Healthcare use and expenditure for diabetes in Bangladesh. BMJ Glob Health 2017;2:e000033.

30. Sal-sabil T, Islam A, Shariful Islam S. Risk Factors for Type 2 Diabetes in Bangladesh: A Systematic Review. J Diabetol $2016 ; 7: 5$.

31. Shariful Islam SM, Lechner A, Ferrari U, et al. Mobile phone use and willingness to pay for SMS for diabetes in Bangladesh. J Public Health (Oxf) 2016;38:163-9.

32. Islam SMS, Tabassum R. Implementation of information and communication technologies for health in Bangladesh. Bull World Health Organ 2015;93:806-9.

33. Anand S, Bradshaw C, Prabhakaran D. Prevention and management of CVD in LMICs: why do ethnicity, culture, and context matter? BMC Med 2020;18:7.

34. Islam SMS, Purnat TD, Phuong NTA, et al. Non-Communicable Diseases (NCDs) in developing countries: a symposium report. Global Health 2014;10:81-8.

35. Khetan AK, Purushothaman R, Chami T, et al. The effectiveness of community health workers for CVD prevention in LMIC. Global Heart 2017;12:233-43.e6.

36. Islam SM, Cartledge S, Karmakar C, et al. Validation and acceptability of a cuffless wrist-worn wearable blood pressure monitoring device among users and healthcare professionals: A mixed-method study. JMIR Mhealth Uhealth $2019 ; 7:$ e14706.

37. Islam S, Tabassum R, Liu Y, et al. The role of social media in preventing and managing non-communicable diseases in low-andmiddle income countries: Hope or Hype? Health Policy Technol 2019;8:97-101. 


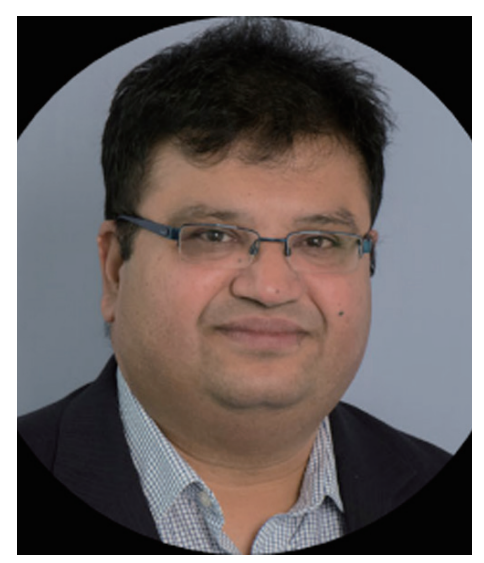

Sheikh Mohammed Shariful Islam

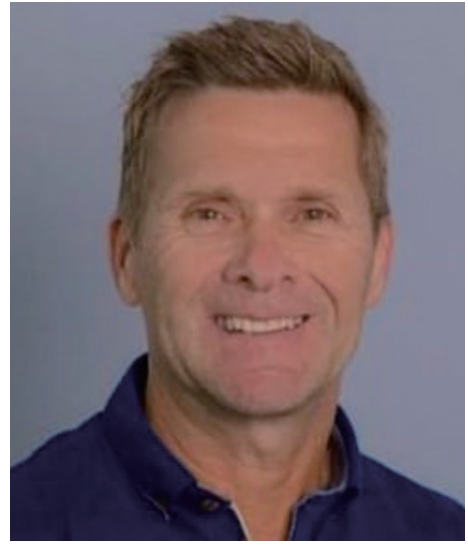

Ralph Maddison

Sheikh Mohammed Shariful Islam, MBBS, MPH, PhD, FESC

(Email: shariful.islam@deakin.edu.au)

Ralph Maddison, PhD

(Email: ralph.maddison@deakin.edu.au)

Institute for Physical Activity and Nutrition, Deakin University, Geelong, Australia Received: 06 August 2020; Accepted: 28 August 2020; Published: 20 July 2021.

doi: $10.21037 / \mathrm{mHealth}-2020-6$

View this article at: http://dx.doi.org/10.21037/mHealth-2020-6

doi: $10.21037 / \mathrm{mHealth}-2020-6$

Cite this article as: Islam SMS, Maddison R. Digital health approaches for cardiovascular diseases prevention and management: lessons from preliminary studies. mHealth 2021;7:41. 